\title{
Application of the '5-2-1' screening criteria in advanced Parkinson's disease: interim analysis of DUOGLOBE
}

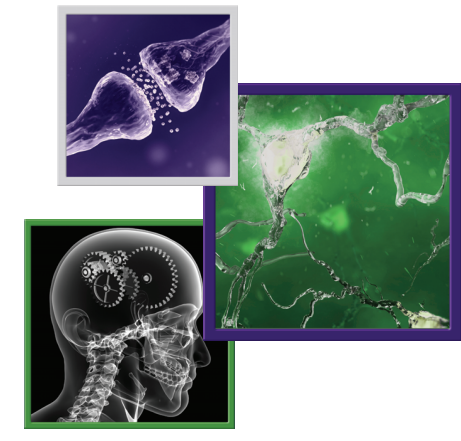

Jason Aldred*,1 (iD), Marieta Anca-Herschkovitsch², Angelo Antonini ${ }^{3}$, Ovidiu Bajenaru ${ }^{4}$, Lars Bergmann $^{5}$, Paul Bourgeois ${ }^{6}$, Esther Cubo ${ }^{7}$, Thomas L Davis ${ }^{8}$, Robert lansek ${ }^{9}$, Norbert Kovács $^{10}$, Pavnit Kukreja ${ }^{5}$, Koray Onuk ${ }^{5}$, Francesco E Pontieri ${ }^{11}$, Weining Robieson ${ }^{5}$, Mustafa S Siddiqui ${ }^{12}$, Mihaela Simu ${ }^{13}$, David G Standaert ${ }^{14}$ \& K Ray Chaudhuri ${ }^{15}$

${ }^{1}$ Selkirk Neurology \& Inland Northwest Research, Spokane, 610 S Sherman St \#201 \& 208, Spokane, WA 99202, USA

${ }^{2}$ Department of Neurology, Edith Wolfson Medical Center Affiliated to Tel Aviv University, 62 Ha-Lokhamim St, Holon, Israel

${ }^{3}$ Department of Neuroscience, Padua University, 5 - 35128 Padova, Padua, Veneto, Italy

${ }^{4}$ Department of Neurology, University of Medicine \& Pharmacy "Carol Davila”, Bulevardul Eroii Sanitari 8, Bucharest, Romania

${ }^{5}$ AbbVie, Inc., 1 Waukegan Rd, North Chicago, IL 60064, USA

${ }^{6}$ Department of Neurology AZ Groeninge, President Kennedylaan 4, Kortrijk, Belgium

${ }^{7}$ Neurology Department, Hospital Universitario Burgos, Av. Islas Baleares, 3, Burgos, Spain

${ }^{8}$ Department of Neurology, Vanderbilt University Medical Center, 1211 Medical Center Dr, Nashville, TN 37232, USA

${ }^{9}$ Kingston Centre, Monash Health, 400 Warrigal Rd, Melbourne, Victoria, Australia

${ }^{10}$ Department of Neurology, University of Pecs, 48 -as tér 1 , Pecs, Hungary

${ }^{11}$ Department of Neurological Sciences, Sapienza University of Rome, Piazzale Aldo Moro, 5, Rome, Italy

${ }^{12}$ Department of Neurology, Wake Forest School of Medicine, 475 Vine St, Winston Salem, NC 27101, USA

${ }^{13}$ Department of Neurology, Victor Babes University of Medicine \& Pharmacy, Piaţa Eftimie Murgu 2, Timisoara, Romania

${ }^{14}$ Department of Neurology, University of Alabama at Birmingham, 1720 University Blvd, Birmingham, AL 35294, USA

${ }^{15}$ Parkinson Foundation International Centre of Excellence, King's College \& King's College Hospital, Denmark Hill, London, UK *Author for correspondence: jaldred@selkirkneurology.com

\section{Practice points}

- Application of simple criteria to identify advanced Parkinson's disease (PD) is important because early identification of advanced PD allows doctors to adjust treatment, leading to better symptom control and improved quality of life.

- A group of experts proposed that fulfilling at least one of the '5-2-1 criteria' (taking levodopa by mouth at least five times a day, having at least $2 \mathrm{~h}$ of the day with 'Off' symptoms, or having at least $1 \mathrm{~h}$ of troublesome, uncontrolled, muscle movements (also known as dyskinesia) suggests advanced PD.

- Patients meeting at least one of the 5-2-1 criteria may also be candidates for advanced therapies, such as continuous infusion of levodopa-carbidopa intestinal gel, continuous administration of subcutaneous apomorphine, or deep brain stimulation.

- The multicountry long-term DUOGLOBE study assessed long-term effectiveness and safety of continuous administration of levodopa-carbidopa intestinal gel.

- At enrollment, almost all patients with physician-identified advanced PD in the DUOGLOBE study met at least one of the 5-2-1 criteria, and the majority (68\%) met two or more of the 5-2-1 criteria.

- Patients showed improvement in motor and nonmotor symptoms following treatment with levodopa-carbidopa intestinal gel.

- As part of the physician's assessment, using the 5-2-1 criteria may be an objective way to identify patients with advanced PD using simple and reproducible measures.

Aim: A Delphi expert consensus panel proposed that fulfilling $\geq 1$ of the '5-2-1 criteria' ( $\geq$ five-times daily oral levodopa use, $\geq$ two daily hours with 'Off' symptoms or $\geq$ one daily hour with troublesome dyskinesia) suggests advanced Parkinson's disease (PD). Patients \& methods: DUOdopa/Duopa in Patients with Advanced PD - a GLobal OBservational Study Evaluating Long-Term Effectiveness (DUOGLOBE) - is a single-arm, postmarketing, observational, long-term effectiveness study of levodopa-carbidopa intestinal gel (LCIG) for advanced PD. Results: This 6-month interim analysis $(n=139)$ affirms that most $(98 \%)$ enrolled patients fulfill $\geq 1$ of the 5-2-1 criteria. These patients responded favorably to LCIG treatment. Safety was consistent with other LCIG studies. Conclusion: In advanced PD patients, the 5-2-1 criteria generally aligns with clinician assessment.

Clinical Trial Registration: NCT02611713 (ClinicalTrials.gov) 
First draft submitted: 21 April 2020; Accepted for publication: 30 July 2020; Published online: 2 September 2020

Keywords: 5-2-1 • criteria for advanced PD $\bullet$ dyskinesia $\bullet$ LCIG $\bullet$ levodopa $\bullet$ levodopa-carbidopa intestinal gel $\bullet$ off time • Parkinson's disease

Levodopa, a dopamine precursor, is a key medication in the standard of care for Parkinson's disease (PD). Although oral treatment with levodopa is highly effective, there are shortcomings that become apparent over time [1]. Doserelated motor and nonmotor fluctuations often follow the dosing cycle, with patients demonstrating 'Off' related deterioration in motor function and emergence of nonmotor symptoms as the medication wears off $[2,3]$. As PD progresses, patients typically require more frequent levodopa dosing as the therapeutic window narrows. Dose fractioning of five or more times daily and troublesome 'Off' periods more than $1-2 \mathrm{~h} /$ day are critical indicators that referral to a specialist may be warranted [4].

Patients with advanced PD are required to have individual, customized therapy to manage symptoms, including nonmotor symptoms, dyskinesia and 'Off' time [5]. When modified oral regimens no longer adequately manage PD symptoms, therapies such as continuous infusion formulations of levodopa, subcutaneous apomorphine infusion and deep brain stimulation are available alternatives for consideration [4]. Levodopa-carbidopa intestinal gel (LCIG) is continuously delivered via percutaneous endoscopic gastrostomy with a jejunal extension tube and a portable pump. Results from several Phase III, observational and comparative studies have demonstrated significant improvements in motor function, nonmotor symptoms and quality of life in patients with advanced PD who are treated with LCIG [6-15]. Continuous subcutaneous infusion of apomorphine was shown in multiple open-label studies to reduce 'Off' time, extend 'On' time and improve disability and nonmotor symptoms [16-18]. Results from a double-blind, placebo-controlled study with apomorphine demonstrated significant reduction in 'Off' time and time without troublesome dyskinesia (TSD) [19]. Effective surgical procedures for advanced PD also exist, such as deep brain stimulation of the subthalamic nucleus and globus pallidus internus, which has been shown to consistently improve motor fluctuations and dyskinesia [16,20-24]. Deep brain stimulation has also been evaluated in patients with less advanced disease and has been shown to provide benefits comparable to a control group receiving the best medical treatment via drug therapy $[14,16,25,26]$.

Efforts to establish simple criteria for the early identification of suspected advanced PD and identification of patients who would benefit from infusion or surgical therapies have been ongoing for some years. The absence of a biomarker, diagnostic test, or gold standard index makes defining the stage of advanced PD challenging, which impacts the ability to optimize therapies [4]. A Delphi expert consensus panel proposed several features across motor, nonmotor and functional-impact domains that might be useful to identify advanced PD. The chosen objective motor criteria (5-2-1 criteria) included using oral levodopa at least five times per day, having at least $2 \mathrm{~h}$ of the day with 'Off' symptoms, or at least $1 \mathrm{~h}$ of the day with TSD [4]. The 5-2-1 criteria may be useful to aid in the identification of suspected advanced PD patients who are uncontrolled with oral/transdermal therapies and may benefit from advanced treatments.

DUOGLOBE (DUOdopa/Duopa in Patients with Advanced Parkinson's Disease - a GLobal OBservational Study Evaluating Long-Term Effectiveness) is a 3-year, follow-up, observational, multicountry study. This post hoc analysis of an interim DUOGLOBE dataset was conducted to evaluate if patients identified by experienced clinicians as having advanced PD met the 5-2-1 criteria. DUOGLOBE also assessed the relationship of the 5-2-1 criteria to effectiveness and safety outcomes of LCIG treatment during routine care. The population was analyzed across four subgroups, divided as patients meeting the five or more times a day oral levodopa dosing criterion, two or more hours of 'Off' time criterion, one or more hours a day TSD criterion and those patients who met all three of the 5-2-1 criteria.

\section{Patients \& methods}

Study design \& treatment

DUOGLOBE is a global, multicountry, single-arm, postmarketing observational analysis of the long-term effectiveness of LCIG in patients with advanced PD (NCT02611713; Supplementary Figure 1).

\section{Patients}

Patients were included in the DUOGLOBE study if they were LCIG-naive at the start of the study and eligible to receive LCIG therapy in accord with the approved local LCIG product label for the region where they were enrolled 
in the study (Label outside of the USA: LCIG is indicated for the treatment of advanced levodopa-responsive Parkinson's disease with severe motor fluctuations and hyper-/dyskinesia when available combinations of Parkinson medicinal products have not given satisfactory results. Label in the USA: LCIG is indicated for the treatment of motor fluctuations in patients with advanced Parkinson's disease). In addition, the physician needed to make the decision to treat the patient with LCIG before the patient was approached to participate in the study and the patient had to provide written informed consent. Patients were excluded from consideration if they had any condition included in the contraindications section of the approved local LCIG label, had current treatment with continuous apomorphine infusion, had a score $<24$ on the Mini-Mental State Examination, had participated in a concurrent interventional clinical trial or exhibited a lack of motivation or insufficient language skills to complete study questionnaires. Patients with history of previous surgery for PD (such as deep-brain stimulation or cell transplantation) were also excluded. However, in 2017 an amendment (only applicable to patients enrolled in the USA) allowed for inclusion of patients who had previous surgery for PD.

\section{Assessments}

Determination of patients' fulfillment of 5-2-1 criteria included frequency of daily levodopa intake, measurement of daily 'Off' time as reported by the patient and time spent with TSD as reported by the patient. TSD was defined as dyskinesia severity (Unified Parkinson's Disease Rating Scale [UPDRS] part IV item 33) with a score of 2, 3 or 4 (mild, moderate or severe) as defined in Supplementary Table 1.

\section{Effectiveness assessments}

Effectiveness in this interim analysis was evaluated from baseline to 6 months follow-up using 'Off' time as reported by the patient and by responses on the Unified Dyskinesia Rating Scale (UDysRS), the Non Motor Symptom Scale (NMSS), the Parkinson's Disease Questionnaire (PDQ-8), the UPDRS part II (activities of daily living) and the Modified Caregiver Strain Index (MCSI).

\section{Safety assessments}

Safety outcomes were assessed based on serious adverse events (AEs), pregnancies and product complaints that were monitored and reported by the physician.

\section{Statistical analysis}

This was a post hoc analysis of the first interim dataset of the DUOGLOBE study. Patients were stratified into subgroups who met all, one or more and each individual 5-2-1 criteria at baseline. Baseline demographics and disease characteristics were analyzed using descriptive statistics. For effectiveness outcomes, statistical comparisons within each group were assessed using a one-sample $t$-test with a p-value $<0.05$ being the cut off level for significance. As this was a post hoc analysis of nonrandomized groups that could have underlying differences in observed or unobserved baseline characteristics, statistical comparisons between groups were not performed.

\section{Results}

\section{Patients}

Patients were enrolled in Australia, Belgium, Hungary, Israel, Italy, Romania, Slovenia, Spain, the UK and the USA. Of the 139 enrolled patients in this interim dataset, 81 had all 5-2-1 criteria status captured at baseline, had 6 month follow-up data and were included in the interim analysis. Most patients (98\%) fulfilled at least one of the 5-2-1 criteria. Over $90 \%$ of patients $(n=73)$ reported having two or more hours of 'Off' time daily at baseline (Figure 1A). More than half (58\%) of patients were taking oral levodopa five-times a day or more $(\mathrm{n}=47)$ and $38 \%$ reported experiencing at least $1 \mathrm{~h}$ of TSD daily $(\mathrm{n}=31)$. The majority $(68 \%)$ of patients fulfilled two or more of the 5-2-1 criteria and 20\% fulfilled all three (Figure 1B). Patients who met none of the 5-2-1 criteria $(\mathrm{n}=2)$ or reported less than $2 \mathrm{~h}$ a day of 'Off' time $(\mathrm{n}=8)$ had insufficient sample sizes for separate analysis of the effectiveness outcomes. These patients were included in the effectiveness and safety outcomes analysis for LCIG treatment.

\section{Patients using more than five versus less than five daily doses of oral levodopa}

When stratified by baseline frequency of oral levodopa dosing, patient characteristics across groups were similar, with both groups comprised mostly of males around age 70 years with approximately 11 years since being diagnosed 
(A)
Oral levodopa
$\geq$ five times/day
$n=47$

$\mathrm{n}=47$
$\mathbf{( 5 8 \% )}$

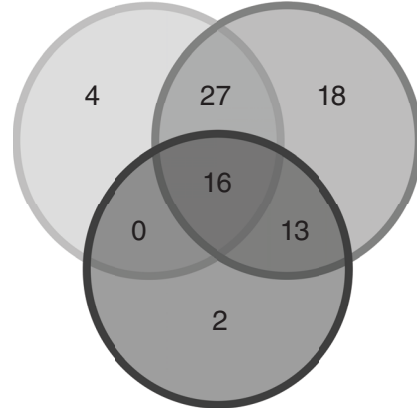

$\geq 1$ hour

Troublesome dyskinesia

(or UPDRS item 33 score $\geq 2$ )

$\mathrm{n}=31$

$(38 \%)$ $\geq 2$ hours

"Off" time

$\mathrm{n}=73$

$(90 \%)$

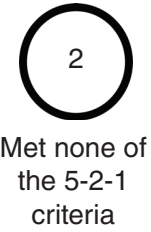

$\mathrm{N}=81$
(B)

Oral tablet intake
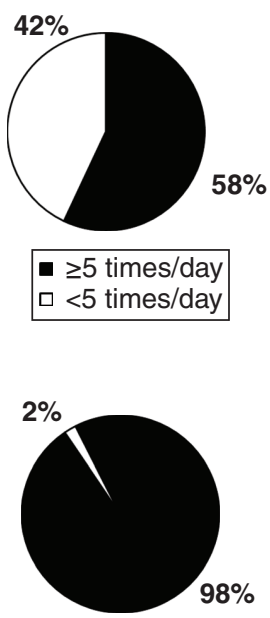

- Met $\geq 1$ $\square$ Met none

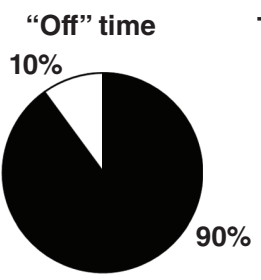

- $\geq 2$ hours/day ᄃ $<2$ hours/day

\section{5-2-1 criteria}

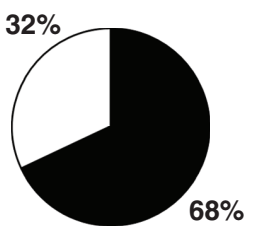

- Met $\geq 2$

$\square$ Met $<2$
Troublesome dyskinesia
$62 \%$
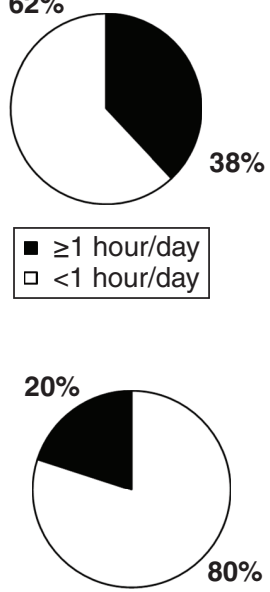

- Met 3 (all)

$\square$ Met $<3$ (not all)
Figure 1. DUOdopa/Duopa in Patients with Advanced Parkinson's Disease - a Global Observational Study Evaluating Long-Term Effectiveness study population who met each individual, any (one or more) and all of the 5-2-1 criteria for advanced Parkinson's disease. (A) Distribution of study population at baseline. (B) Each graph represents the analysis population $(n=81)$ and if those patients met (black) or did not meet (white) the 5-2-1 criteria for advanced PD in each subgroup. PD: Parkinson's disease; UPDRS: Unified Parkinson's Disease Rating Scale.

with PD (Supplementary Table 2). After 6 months of LCIG therapy, both groups exhibited significant reductions from baseline in 'Off' time ( $p<0.001$ for both), UDysRS ( $p<0.01$ for five or more times a day of oral levodopa and $\mathrm{p}<0.001$ for five or more times a day of oral levodopa) and NMSS scores ( $<<0.001$ for both). PDQ-8 summary index scores were significantly reduced in the five or more times a day oral levodopa group $(\mathrm{p}<0.001$; Figure 2). No significant improvements were seen in UPDRS part II scores or mean MCSI scores.

\section{Patients experiencing $\geq 2 \mathrm{~h}$ a day versus $<2 \mathrm{~h}$ a day 'Off' time}

Patients who had two or more hours a day of 'Off' time at baseline were on average 68.6 years of age with PD of 11 -years' duration. Average 'Off' time was $6.3 \mathrm{~h}$. No analysis was performed in the group of patients who had $<2 \mathrm{~h}$ a day of 'Off' time because of the small group size (Supplementary Table 3). Patients with at least $2 \mathrm{~h}$ of 'Off' time a day represent nearly the full study population, so effectiveness data in this group provides the closet estimation of the overall interim results. After 6 months of LCIG therapy, patients with two or more hours a day baseline 'Off' time exhibited significant reductions in 'Off' time ( $p<0.001)$, UDysRS $(\mathrm{p}<0.001)$, NMSS $(\mathrm{p}<0.001)$, PDQ-8 summary index $(\mathrm{p}<0.01)$ and MCSI scores $(\mathrm{p}<0.05)$ (Figure 3$)$. The changes from baseline in UPDRS part II score were not significant after 6 months for the group experiencing at least $2 \mathrm{~h}$ of 'Off' time daily.

Patients experiencing one or more hours a day versus less than $1 \mathrm{~h}$ a day TSD

When stratified by baseline TSD, patient characteristics across groups were similar, including UPDRS part II and PDQ-8 summary index scores, but with notable differences including more dyskinesia time and a higher UDysRS 

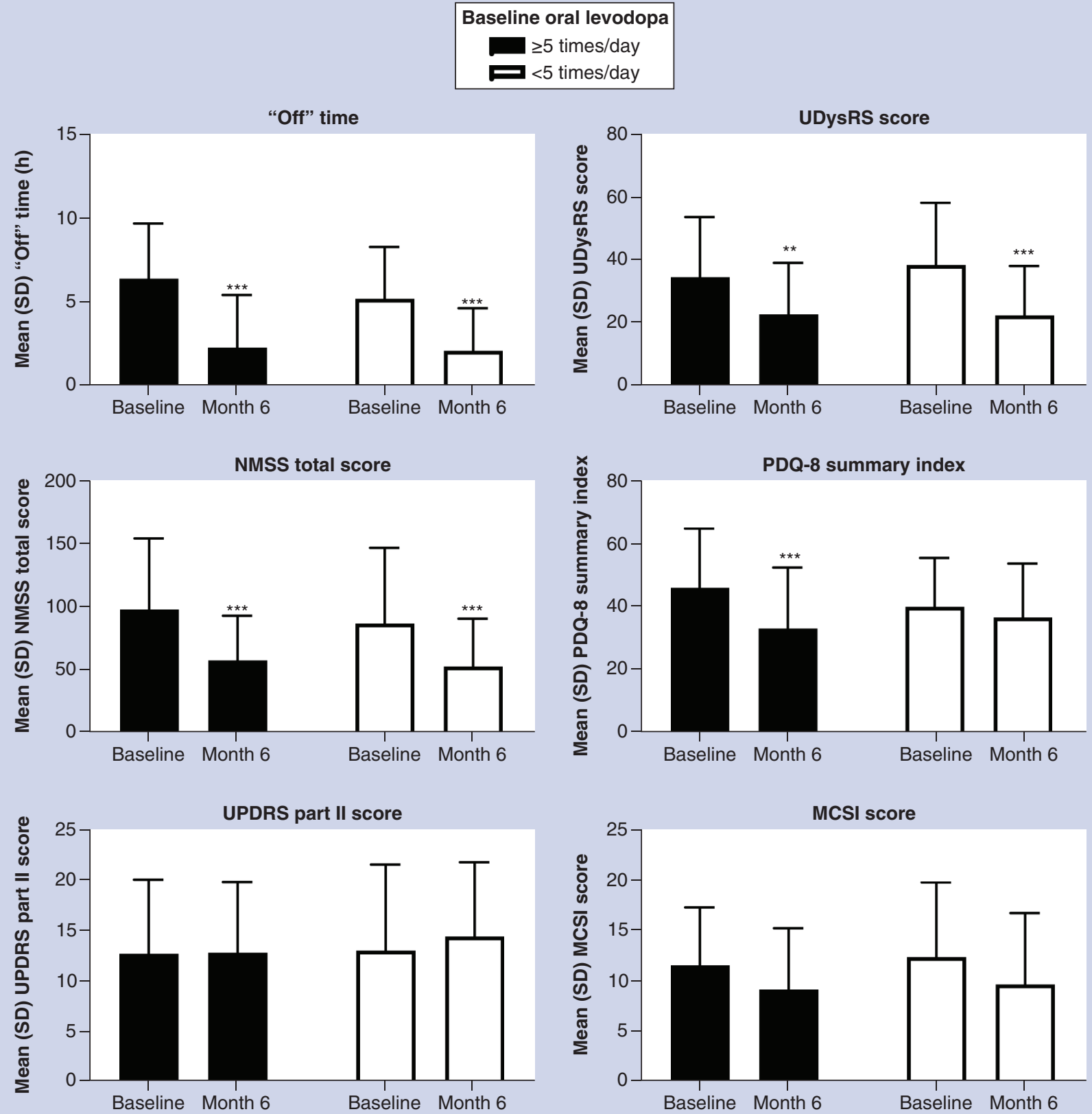

Figure 2. Effectiveness outcomes in patients stratified by baseline oral levodopa dosing frequency.

$* * *$ Statistically significant at $\mathrm{p}<0.001 ; * *$ Statistically significant at $\mathrm{p}<0.01$.

MCSI: Modified Caregiver Strain Index; NMSS: Non Motor Symptoms Scale; PDQ-8: Parkinson's Disease Questionnaire-8; SD: Standard deviation; UDysRS: Unified Dyskinesia Rating Scale.

score in patients with one or more hours a day TSD, although no statistical comparison was made (Supplementary Table 4). After 6 months of LCIG therapy, both groups exhibited significant reductions in 'Off' time and NMSS scores $(\mathrm{p}<0.001)$. Patients experiencing at least $1 \mathrm{~h}$ of TSD a day also had significantly reduced UDysRS scores at 6 months $(\mathrm{p}<0.001)$, as did patients experiencing less than $1 \mathrm{~h}$ of TSD $(\mathrm{p}<0.01)$. PDQ 8 summary index scores were significantly reduced in the one or more hours a day TSD group $(\mathrm{p}<0.01)$ (Figure 4). MCSI scores were significantly reduced $(\mathrm{p}<0.05)$ in patients who experienced TSD $<1$ h a day. No significant differences were found in UPDRS part II scores after 6 months for either group. 


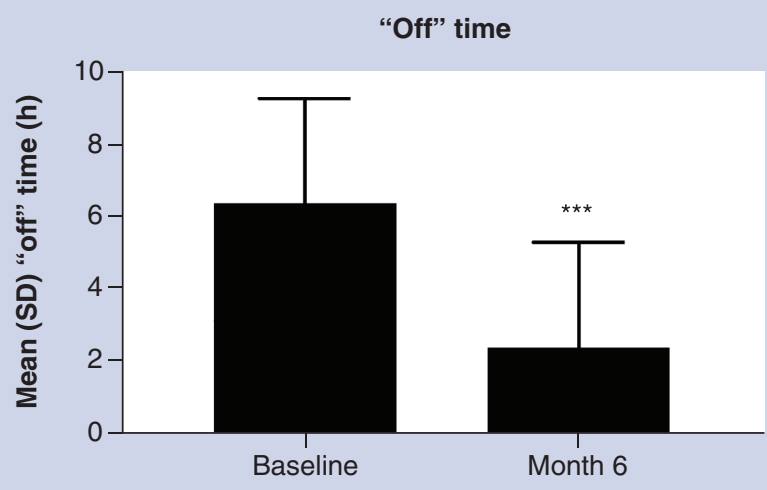

NMSS total score

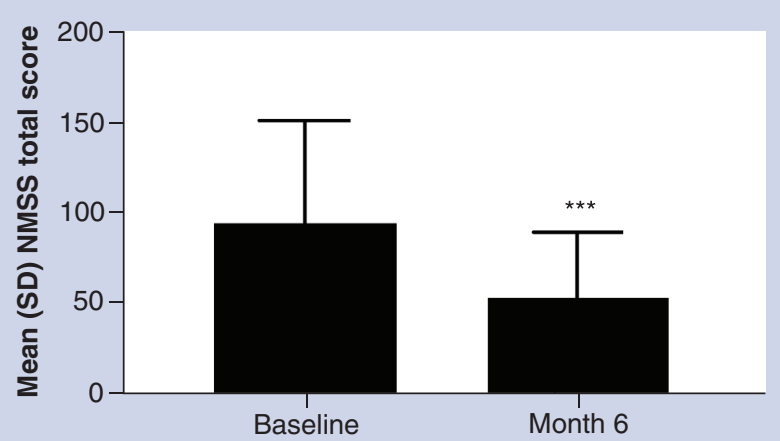

UPDRS part II score

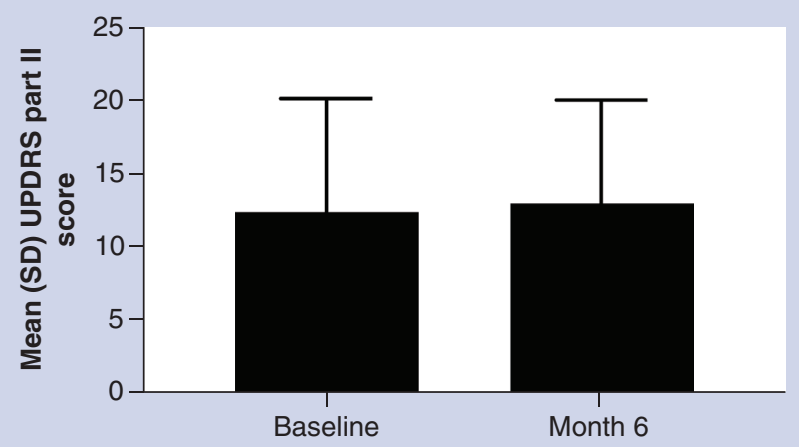

UDysRS score

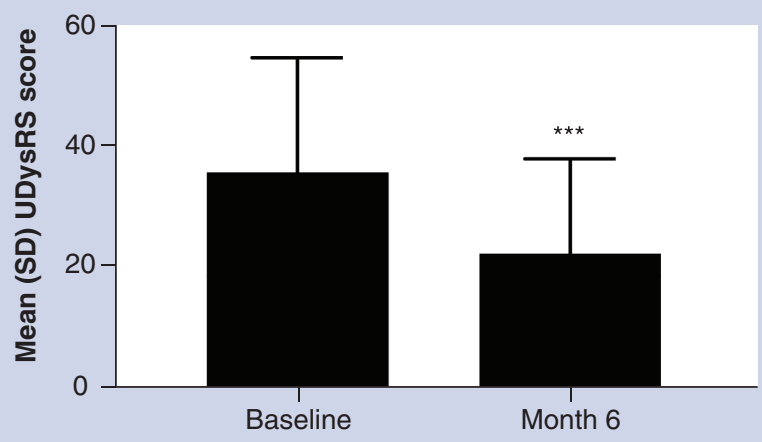

PDQ-8 summary index

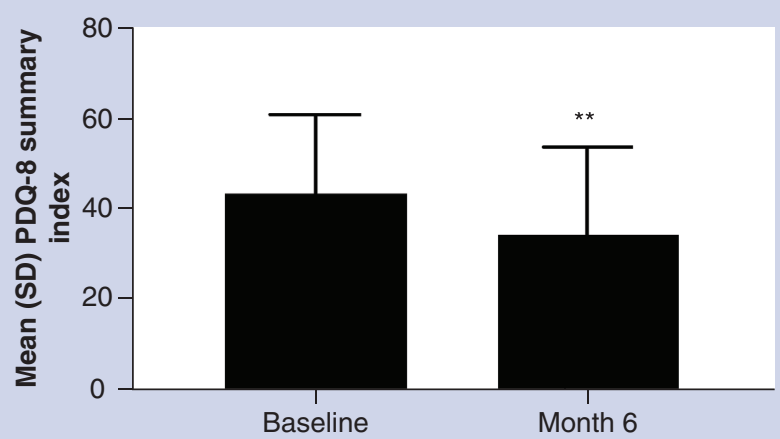

MCSI score

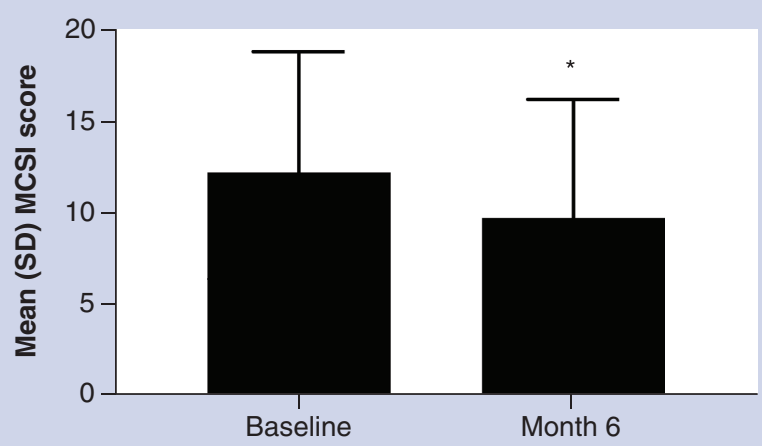

Figure 3. Effectiveness outcomes in patients with baseline 'Off' time $\geq 2 \mathrm{~h} /$ day. Patients who reported $<2 \mathrm{~h} /$ day of 'Off' time ( $\mathrm{n}=8$ ) had insufficient sample sizes for analysis of the effectiveness outcomes. ${ }^{* *}$ Statistically significant at $p<0.001$; **Statistically significant at $\mathrm{p}<0.01$; *Statistically significant at $\mathrm{p}<0.05$.

MCSI: Modified Caregiver Strain Index; NMSS: Non Motor Symptoms Scale; PDQ-8: Parkinson's Disease Questionnaire-8; SD: Standard deviation; UDysRS: Unified Dyskinesia Rating Scale.

Patients fulfilling all of the 5-2-1 criteria versus those not meeting all criteria

When stratified by baseline fulfillment of the 5-2-1 criteria, patient characteristics across groups were similar (Table 1). Patients who fulfilled all the 5-2-1 criteria appeared to have the highest baseline burden in terms of 'Off' time, UPDRS part II, UDysRS, NMSS, PDQ-8 summary index and MCSI scores. After 6 months of LCIG therapy, both groups exhibited significant reductions in most effectiveness measurements $(\mathrm{p}<0.001$ for the 'Off' time subgroup not fulfilling all criteria and both UDysRS subgroups; $\mathrm{p}<0.01$ for the 'Off' time and NMSS subgroups fulfilling all criteria; $\mathrm{p}<0.001$ for NMSS in the group not fulfilling all criteria; and $\mathrm{p}<0.05$ for PDQ-8 and MCSI for the subgroup not fulfilling all criteria; Figure 5). MCSI scores were significantly reduced for 


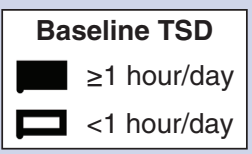

"Off" time

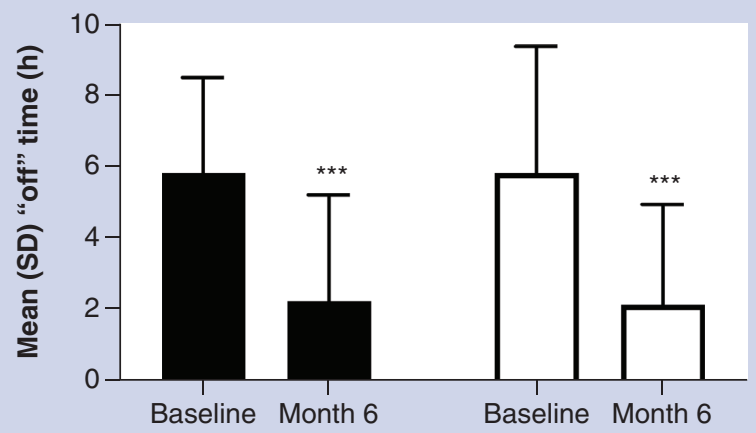

NMSS total score

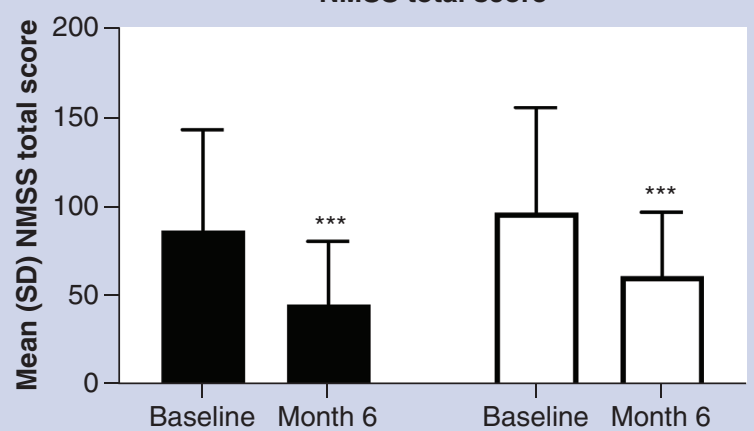

UPDRS part II score

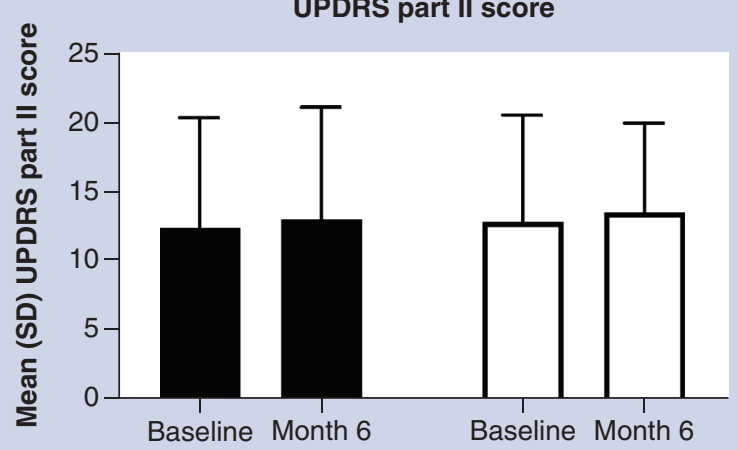

UDysRS score

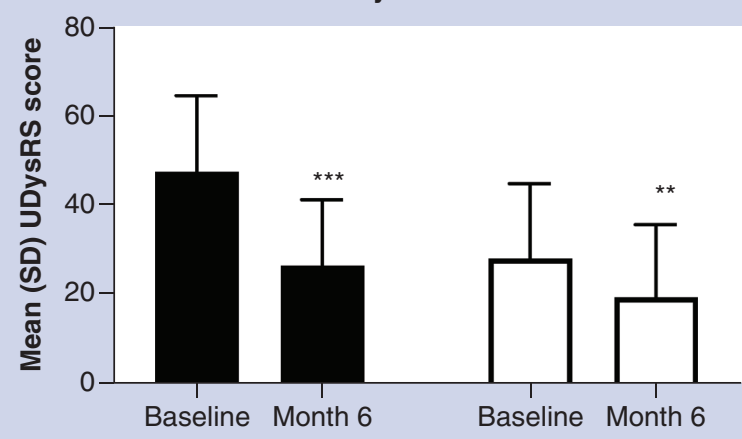

PDQ-8 summary index
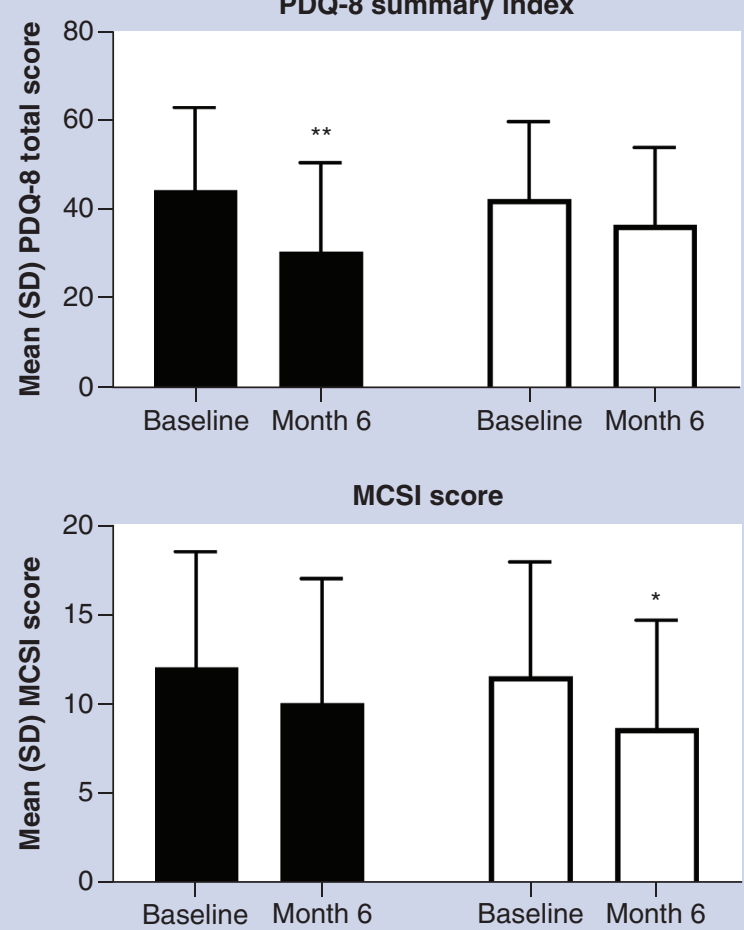

Figure 4. Effectiveness outcomes in patients stratified by baseline troublesome dyskinesia.

$* * *$ Statistically significant at $p<0.001 ; * *$ Statistically significant at $p<0.01$; $*$ Statistically significant at $p<0.05$.

MCSI: Modified Caregiver Strain Index; NMSS: Non Motor Symptoms Scale; PDQ-8: Parkinson's Disease Questionnaire-8; SD: Standard deviation; TSD: Troublesome dyskinesia; UDysRS: Unified Dyskinesia Rating Scale.

the group of patients who did not meet all criteria $(\mathrm{p}=0.018)$. There was no significant difference for either group in UPDRS part II scores.

\section{Safety assessments}

The safety dataset of this first interim analysis included 139 patients, but 81 patients had full baseline data on the 5-2-1 criteria. Serious AEs related to LCIG occurred at a similar rate in each group (Table 2). The most common AEs occurring in two or more patients in each group were decubitus ulcer, device occlusion, femoral neck fracture, general physical health deterioration, pneumonia, pneumoperitoneum See Table 2 for groups with 
Table 1. Baseline characteristics stratified by baseline fulfillment of 5-2-1 criteria.

\begin{tabular}{|c|c|c|}
\hline \multirow[t]{2}{*}{ Parameter } & \multicolumn{2}{|c|}{ Mean (SD) ${ }^{\dagger}$} \\
\hline & Met all 5-2-1 criteria $(n=16)$ & Did not meet all $(n=65)$ \\
\hline \multicolumn{3}{|l|}{ Sex, n (\%) } \\
\hline Female & $4(25)$ & $21(32)$ \\
\hline Male & $12(75)$ & $44(67.7)$ \\
\hline Age in years & $67.6(7.83)$ & $69.8(8.63)$ \\
\hline Time since diagnosis in years & $12.2(5.50)$ & $11.1(4.72)$ \\
\hline Hoehn and Yahr stage $\ddagger$ & $3.0(1.0,5.0)^{\S}$ & $3.0(1.0,5.0) \mathbb{I}$ \\
\hline 'Off' time in hours & $6.6(2.10)$ & $5.5(3.33)$ \\
\hline Dyskinesia time in hours & $4.6(2.34)$ & $4.0(3.86)$ \\
\hline UDysRS score & $50.8(13.31)$ & $31.4(20.47)$ \\
\hline NMSS total score & $108.1(51.00)$ & $92.0(58.16)$ \\
\hline PDQ-8 summary index & $49.8(16.60)$ & $42.2(17.12)$ \\
\hline UPDRS part II score & $14.0(7.22)$ & $13.5(8.08)$ \\
\hline MCSI score & $12.4(5.62)$ & $11.3(6.72)$ \\
\hline \multicolumn{3}{|c|}{$\begin{array}{l}\dagger \text { Data are presented as mean (SD), unless otherwise noted. } \\
\ddagger \text { Median (range). } \\
\S_{\mathrm{n}}=15 \\
\mathbb{I}_{\mathrm{n}}=64\end{array}$} \\
\hline
\end{tabular}

\begin{tabular}{|c|c|c|c|c|c|c|c|}
\hline & \multicolumn{2}{|c|}{ Daily oral levodopa frequency } & \multirow{2}{*}{$\begin{array}{l}\text { 'Off' time } \\
\geq 2 \mathrm{~h} / \text { day } \\
(\mathrm{n}=73)\end{array}$} & \multicolumn{2}{|c|}{ Troublesome dyskinesia } & \multicolumn{2}{|c|}{ 5-2-1 criteria } \\
\hline & $\begin{array}{l}\geq \text { Five-times/day } \\
(n=47)\end{array}$ & $\begin{array}{l}<\text { Five-times/day } \\
(\mathrm{n}=34)\end{array}$ & & $\begin{array}{l}\geq 1 \mathrm{~h} / \text { day } \\
(\mathrm{n}=31)\end{array}$ & $\begin{array}{l}<1 \mathrm{~h} / \text { day } \\
(\mathrm{n}=50)\end{array}$ & All $(n=16)$ & $\begin{array}{l}\text { Not all } \\
(n=65)\end{array}$ \\
\hline Any serious $A E$ & $19(40)$ & $9(26.5)$ & $24(33)$ & $8(26)$ & $20(40)$ & $4(25)$ & $24(37)$ \\
\hline Any serious AE possibly related to $L C I G$ & $6(13)$ & $4(12)$ & $8(11)$ & $3(10)$ & $7(14)$ & $2(13)$ & $8(12)$ \\
\hline Deaths & $2(4)$ & $2(6)$ & $4(6)$ & $3(10)$ & $1(2)$ & $1(6)$ & $3(5)$ \\
\hline \multicolumn{8}{|c|}{ Most common AEs (occurring in $\geq$ two patients/group) } \\
\hline Decubitus ulcer & $2(4)$ & - & $2(3)$ & $1(3)$ & $1(2)$ & $1(6)$ & $1(2)$ \\
\hline Device occlusion & $1(2)$ & 1 (3) & $1(1)$ & - & $2(4)$ & - & $2(3)$ \\
\hline Femoral neck fracture & $2(4)$ & - & $2(3)$ & $2(7)$ & - & $2(13)$ & - \\
\hline General physical health deterioration & $2(4)$ & - & $2(3)$ & $1(3)$ & $1(2)$ & $1(6)$ & $1(2)$ \\
\hline Pneumonia & $1(2)$ & 1 (3) & $2(3)$ & $2(7)$ & - & $1(6)$ & $1(2)$ \\
\hline Pneumoperitoneum & $2(4)$ & - & $2(3)$ & - & $2(4)$ & - & $2(3)$ \\
\hline Urinary tract infection & $1(2)$ & 1 (3) & $1(1)$ & - & $1(2)$ & - & $1(2)$ \\
\hline Discontinuations & $8(17)$ & $3(9)$ & $11(15)$ & $3(10)$ & $8(16)$ & $1(6)$ & $10(15)$ \\
\hline Discontinuations due to $A E s$ & $4(9)$ & $3(9)$ & $7(10)$ & $2(7)$ & $5(10)$ & 0 & $7(11)$ \\
\hline
\end{tabular}

$\mathrm{n}=1$. Polyneuropathy occurred in one patient (between months 7 and 12). There was one report of 'sensory loss' between months 1 and 6, which may be a description of a neuropathic event. Deaths occurred in at least one patient in each subgroup (2-10\%). One patient death (intestinal obstruction) in the interim dataset was deemed to be possibility related to LCIG. Across all subgroups, $6-17 \%$ of patients discontinued the study. The main reasons for discontinuation were AEs (0-9.5\%), withdrawn consent (0-9\%) and other reasons (5-7\%).

\section{Discussion}

As PD progresses, management of motor and nonmotor symptoms becomes more difficult, particularly as administration of oral medication may provide less consistent symptom control [2,3,16,27,28]. Device-aided therapies, such as continuous subcutaneous apomorphine infusion, continuous LCIG infusion and deep brain stimulation, are used to treat advanced PD in selected patients [4]. However, there are knowledge gaps regarding what therapies are most appropriate and when they should be initiated [4,29]. Earlier identification of these patients is expected to lead 

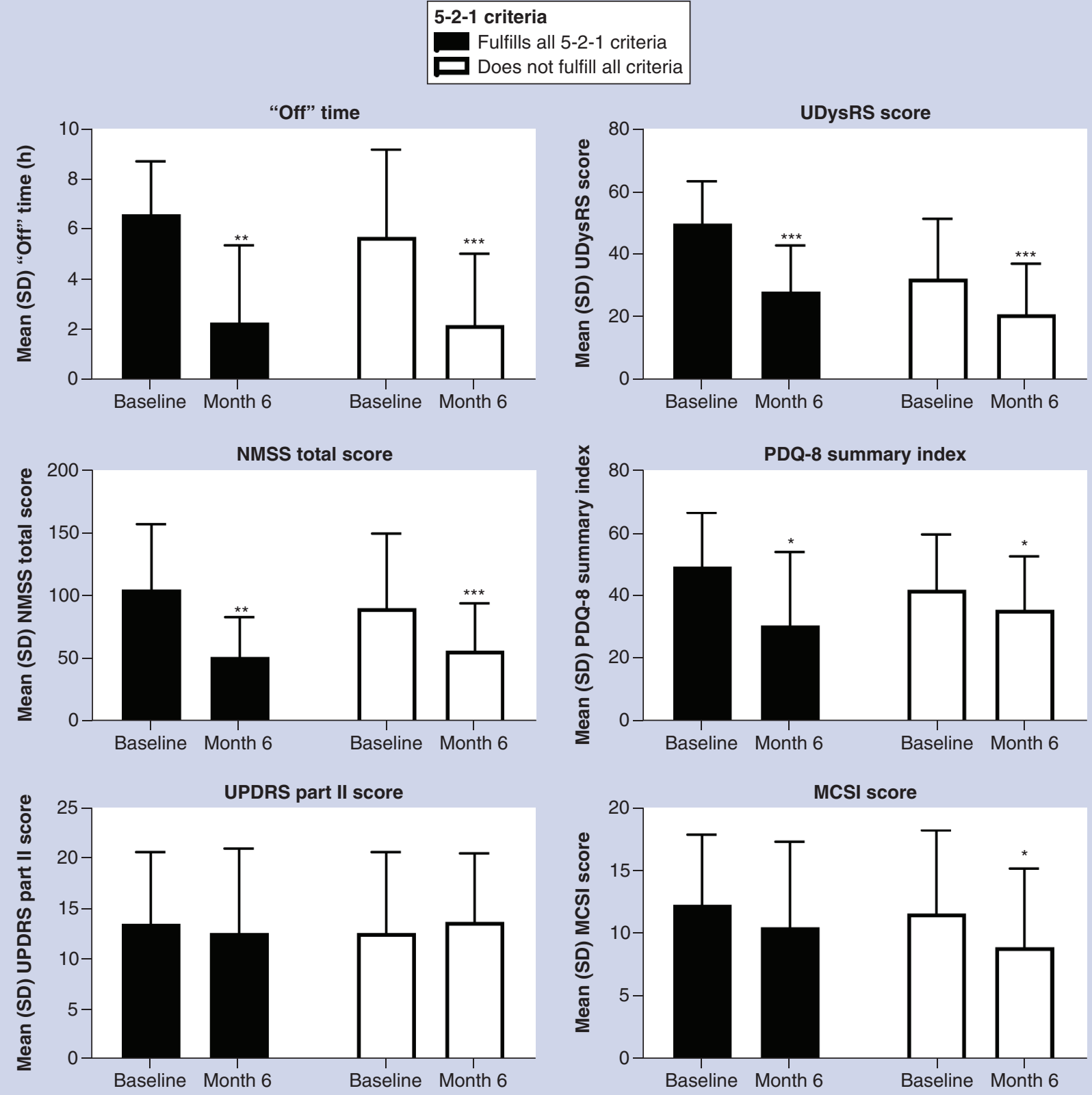

Figure 5. Effectiveness outcomes in patients stratified by fulfillment of all 5-2-1 criteria.

$* * *$ Statistically significant at $p<0.001 ; * *$ Statistically significant at $p<0.01$; ${ }^{*}$ Statistically significant at $p<0.05$. MCSI: Modified Caregiver Strain Index; NMSS: Non Motor Symptoms Scale; PD: Parkinson's disease; PDQ-8: Parkinson's Disease Questionnaire-8; SD: Standard deviation; UDysRS: Unified Dyskinesia Rating Scale.

to improved patient care by earlier initiation of advanced therapies [16]. Criteria to establish the identification of treatable motor symptoms of advanced PD may enable earlier and more uniform recognition of patients who might benefit from advanced therapies, but further validation is needed. Even among specialists in movement disorders, debate exists whether early treatment is based on time for duration of disease versus emergence of clinical first signs of motor fluctuations [30]. The 5-2-1 criteria may help address the clinical gap in timely identification of patients whose symptoms may be uncontrolled with oral medications [4,31]. This criteria is also found in the first section of a recently launched comprehensive screening tool (MANAGE-PD) that has been tested in the USA to screen patients whose PD is no longer controlled with oral medications (www.managepd.com) [14]. 
This study represents the first attempt at using the criteria with a large cohort of patients on an international scale. Interim results demonstrate that almost all patients selected for LCIG by DUOGLOBE investigators on clinical grounds fulfilled at least one of the 5-2-1 criteria. Most commonly, patients had two or more hours of 'Off' time at baseline. Patients treated with LCIG had improvements in 'Off' time, dyskinesia, nonmotor symptoms and quality of life after 6 months. Although the 5-2-1 criteria only identify patients with motor fluctuations, the improvements seen both in some dopaminergic nonmotor symptoms and in quality of life likely occur through the effects of continuously administered levodopa. Patients in this analysis experienced a LCIG safety profile consistent with the safety profile identified in the Phase III trials. Patients who fulfilled all of the 5-2-1 criteria also had the most 'Off' time and highest baseline burden according to baseline scores of UPDRS part II, UDysRS, NMSS, PDQ-8 summary index and MCSI. Results from this study confirm that the 5-2-1 criteria correlate with the established predictors of disease burden and indicate these three relatively simple screening criteria may be useful in clinical practice as part of holistic disease progression assessment and prediction of overall disease burden.

This study is limited by the observational, uncontrolled, open-label design and the fact that these criteria are being applied to patients on LCIG therapy only. The impact of the 5-2-1 criteria on nonmotor symptoms is limited by the fact that two of the three criteria are focused on motor symptoms only. The feasibility of using the 5-2-1 criteria for the selection of patients for other device-aided therapies (e.g., apomorphine subcutaneous infusion and deep brain stimulation) and their impact on effectiveness outcomes needs further evaluation. Section 1 of the emerging MANAGE-PD instrument applies the 5-2-1 screening criteria to determine whether patients are well controlled with oral medications. If patients demonstrate deficiency in any one of the questions in section 1, they are then moved to section 2, where patient eligibility for device-aided therapy is assessed [32]. The MANAGE-PD tool is available online and based on robust quantitative and qualitative data from a panel of leading PD specialists and warrants further validation studies $[16,33]$. Timely management of symptoms in patients with PD using a standardized and validated tool may aid in homogenizing care for patients between PD specialists and general neurologists, including the timing and need for referrals or medication change and reducing the time a patient remains inadequately controlled on oral medications [32].

There are limitations regarding sample size as a few groups in this study were too small to analyze; however, information can still be gleaned from those patients in the larger counterpart subgroup. This study is also limited by including only patients initially screened by expert clinicians as candidates appropriate for treatment with LCIG. Measurement of 'Off' time may have been limited by use of a modified UPDRS III item 39 rather than Hauser diaries and the UPDRS measurement used may cause some ambiguities as it was not the current UPDRS licensed by the Movement Disorder Society. Although application of the 5-2-1 criteria agreed with clinical judgment of advanced PD in patients in this study, results may not be generalizable to all patient populations. The criteria are focused on motor features and did not include nonmotor symptoms, although nonmotor symptoms may be very relevant in choosing therapeutic strategies in treating patients with advanced PD. Exclusion of patients with cognitive impairment limits the application of the study results to that subgroup of patients that may be considered for LCIG therapy.

Clinical scales typically used to assess patients with PD are limited in that they may not capture all patients who have PD that is considered 'advanced'. The UPDRS is a comprehensive assessment of PD; however, a physician with a higher level of expertise (i.e., a movement disorder specialist) may be more adept at using it and use of MDS-UPDRS can be time consuming and expensive [34]. UPDRS scores may also vary over time in patients who have motor fluctuations. Other scales are strictly focused on assessing only specific aspects, such as the Parkinson Fatigue Scale, NMSS and the UDysRS [34-36]. Although, the severe disability denoted by Hoehn and Yahr stage IV and $\mathrm{V}$ [37] usually qualifies a patient as having advanced disease. Elucidating the full clinical picture of advancing $\mathrm{PD}$ requires a holistic approach.

\section{Conclusion}

Results from this interim analysis confirm that the 5-2-1 criteria apply to a population of patients identified by clinicians as having advanced PD in a large, observational, multicountry study. As part of the clinician's assessment and emerging use of the MANAGE-PD tool, applying the 5-2-1 criteria may be a practical and straightforward way to identify patients with advanced PD. While fewer than $20 \%$ of patients in this study met all of the 5-2-1 criteria, all but two (98\%) met at least one criterion, suggesting that meeting any one of the criteria may identify advancing PD. Patients meeting any of the 5-2-1 criteria may also be candidates for advanced therapies, such as continuous infusion with device-aided LCIG, subcutaneous apomorphine or deep-brain stimulation, which could 
offer better control of motor symptom fluctuations. Patients treated with LCIG in this study had improvements in 'Off' time, dyskinesia, nonmotor symptoms and quality of life. Prompt identification of patients with advancing disease is expected to lead to improved patient care by helping recognize those patients who may benefit from referral to specialists in movement disorders and possible initiation of advanced therapies when motor fluctuations become bothersome.

\section{Supplementary infographic}

An infographic accompanies this paper at the end of the references section. To download the infographic that accompanies this paper, please visit the journal website at: www.futuremedicine.com/doi/suppl/10.2217/nmt-2020-0021

\section{Supplementary data}

To view the supplementary data that accompany this paper please visit the journal website at: www.futuremedicine.com/doi/sup $\mathrm{pl} / 10.2217 / \mathrm{nmt}-2020-0021$

\section{Author contributions}

All authors had access to the data and participated in the development, review, critique and approval of the manuscript throughout the editorial process and approved the final manuscript draft submitted for publication. All authors agree to be accountable for all aspects of the work, ensuring the accuracy and integrity of the publication. All named authors meet the International Committee of Medical Journal Editors (ICMJE) criteria for authorship for this article, take responsibility for the integrity of the work as a whole and have given their approval for this version to be published.

All authors critically reviewed this manuscript and provided final approval for publication. J Aldred, A Antonini, K Onuk and KR Chaudhuri contributed to study design and data interpretation. M Anca-Herschkovitsch, O Bajenaru, P Bourgeois, TL Davis, $\mathrm{R}$ lansek, FE Pontieri and MS Siddiqui contributed to data interpretation. L Bergmann and W Robieson contributed to study design, data acquisition, statistical analysis and data interpretation. E Cubo and M Simu contributed to data acquisition. N Kovács contributed to data acquisition and interpretation. P Kukreja and DG Standaert contributed to study design, data acquisition and data interpretation.

\section{Acknowledgments}

The authors thank AbbVie for support of this manuscript. AbbVie and the authors thank the patients who participated in this clinical trial and all study investigators for their contributions.

Financial \& competing interests disclosure

This study was funded by AbbVie, Inc. AbbVie participated in the study design; study research; collection, analysis, and interpretation of data; and writing, reviewing, and approving this manuscript for publication. J Aldred has received honoraria from Abbott Laboratories, AbbVie, Accorda, Allergan, Medtronic, Boston Scientific, Teva, and US World Meds. He has received research funding for studies sponsored by AbbVie, Acadia, Annexon, Amneal/Impax, Biogen, Boston Scientific, Centogene, Denali, Massachusetts General Hospital, Neuroderm, Neurocrine, Northwestern University, Sunovion, Triplet Therapeutics, and Theravance, Inc. He is a scientific advisor for AbbVie, Allergan, and Boston Scientific. M Anca-Herschkovitsch is a consultant for AbbVie Israel. A Antonini has received honoraria for consultancy and speaker-related activities from UCB, Boehringer Ingelheim, AbbVie, Zambon, Bial, Ever Pharma, Neuroderm, Therevance, and Biogen. He receives research support from Chiesi Pharmaceuticals, Lundbeck, Horizon 2020 - PD_Pal Grant 825785, Ministry of Education University and Research (MIUR) Grant ARS01_01081, and Cariparo Foundation. He serves as consultant for Boehringer Ingelheim for legal cases on pathological gambling. He owns Patent WO2015110261-A1 and owns shares from PD Neurotechnology Limited. O Bajenaru received investigator's fees for clinical research from AbbVie and Ipsen, and speaker's fees from AbbVie, Union Chimique Belge, and Ipsen. He is a member of advisory boards at AbbVie. L Bergmann, P Kukreja, K Onuk, and W Robieson are employees of AbbVie, and may hold AbbVie stock and/or stock options. P Bourgeois and R lansek have received honoraria for consulting from AbbVie. E Cubo has received travel grants and speaker-related activities from AbbVie and Boston Scientific. TL Davis has received research support from the Parkinson Foundation and the Peterson Foundation for Parkinsons and honoraria for consulting from AbbVie and Acadia Pharmaceuticals. N Kovács was a study investigator and has received honorarium from AbbVie, Abbott, Boston Scientific, Boehringer Ingelheim, GSK Pharmaceuticals, KRKA, Medtronic, Teva, and UCB Pharma, and has also received honoraria for lecturing at symposia. He has been a consultant for AbbVie, Abbott, KRKA, and Teva. He has received research funding from the Hungarian National Research, Development and Innovation Office; University of Pécs; and Medtronic. FE Pontieri is a member of the faculty at the Sapienza University of Rome and has a clinical practice at Sant'Andrea Hospital in Rome. He is an investigator in studies funded by AbbVie, Zambon, and the Italian Ministry of Health. He 
has served as consultant for or received honoraria from AbbVie, Zambon, FB Health, Bial, and Lundbeck. MS Siddiqui has served on scientific advisory boards for Boston Scientific and AbbVie. He has received research support for clinical trials sponsored by Sunovion Pharma, AbbVie, Boston Scientific, Inc., Biogen MA, Inc., Theravance Biopharma, Michael J Fox / Neuropoint Alliance, Impax Laboratories, and Sun Pharma. M Simu has received honoraria for lecturing at symposia and consultancy services from AbbVie, AOP Orphan, Boehringer Ingelheim, Krka, Merck, Sanofi, Servier Pharma, Teva, and UCB Pharma. DG Standaert is a member of the faculty of the University of Alabama at Birmingham and is supported by endowment and University funds. He is an investigator in studies funded by AbbVie Inc.; Avid Radiopharmaceuticals; the American Parkinson Disease Association; the Michael J. Fox Foundation for Parkinson Research; Alabama Department of Commerce; the Department of Defense; and NIH grants P01NS087997, P50NS108675, R25NS079188, P2CHD086851, P30NS047466, and T32NS095775. He has a clinical practice and is compensated for these activities through the University of Alabama Health Services Foundation. In addition, since January 1, 2019, he has served as a consultant for or received honoraria from Axovant Sciences, Inc., Censa Pharmaceuticals, AbbVie Inc., Grey Matter Technologies, Theravance Inc., the Kennedy Krieger Institute, McGraw Hill Publishers, Sanofi- Aventis, RTI Consultants, Cerevance Inc., Yale University, and Michigan State University. KR Chaudhuri has received honoraria for lectures from AbbVie, Boehringer Ingelheim, Neuroderm, Britannia Pharmaceuticals, Mundipharma, Novartis, Sunovion, UCB Pharma, and Zambon. He has served on advisory boards for AbbVie, Bial, Cynapsus, GKC, Jazz Pharmaceuticals, Lobsor, Medtronic, Novartis, Pfizer, Profile, Stada, Sunovion, UCB Pharma, and Zambon. He has received investigator-initiated grants from AbbVie, Bial, Britannia Pharmaceuticals, GKC, and UCB Pharma, and academic grants from the European Union, Horizon 2020, Innovative Medicines Initiative EU, Kirby Laing Foundation, Medical Research Council, National Institute for Health Research, Parkinson's UK, National Parkinson Foundation, and Parkinson's Disease Non-Motor Group.

Medical writing support, funded by AbbVie, was provided by Kelly M Cameron, PhD, Certified Medical Publication Professional ${ }^{T M}$ and Meghan L Thompson, PharmD, PhD, of JB Ashtin, who developed the first draft based on an author-approved outline and assisted in implementing author revisions throughout the editorial process.

Ethical conduct of research

The authors state that they have obtained appropriate institutional review board approval or have followed the principles outlined in the Declaration of Helsinki for all human or animal experimental investigations. In addition, informed consent has been obtained from the all participants involved.

\section{Data sharing statement}

AbbVie is committed to responsible data sharing regarding the clinical trials we sponsor. This includes access to anonymized, individual, and trial-level data (analysis data sets), as well as other information (e.g., protocols and Clinical Study Reports), as long as the trials are not part of an ongoing or planned regulatory submission. This includes requests for clinical trial data for unlicensed products and indications. This clinical trial data can be requested by any qualified researchers who engage in rigorous, independent scientific research, and will be provided following review and approval of a research proposal and Statistical Analysis Plan (SAP) and execution of a Data Sharing Agreement (DSA). Data requests can be submitted at any time and the data will be accessible for 12 months, with possible extensions considered. For more information on the process, or to submit a request, visit the following link: https://www.AbbVie.com/our-science/clinical-trials/clinical-trials-data-and-information-sharing/data-and-infor mation-sharing-with-qualified-researchers.html.

\section{Open access}

This work is licensed under the Attribution-NonCommercial-NoDerivatives 4.0 Unported License. To view a copy of this license, visit http://creativecommons.org/licenses/by-nc-nd/4.0/

\section{References}

Papers of special note have been highlighted as: $\bullet$ of interest; $\bullet \bullet$ of considerable interest

1. Chase TN. Levodopa therapy: consequences of the nonphysiologic replacement of dopamine. Neurology 50(5 Suppl. 5), S17-S25 (1998).

2. Antonini A, Chaudhuri KR, Martinez-Martin P, Odin P. Oral and infusion levodopa-based strategies for managing motor complications in patients with Parkinson's disease. CNS Drugs 24(2), 119-129 (2010).

3. Chaudhuri KR, Poewe W, Brooks D. Motor and nonmotor complications of levodopa: phenomenology, risk factors and imaging features. Mov. Disord. 33(6), 909-919 (2018).

4. Antonini A, Stoessl AJ, Kleinman LS et al. Developing consensus among movement disorder specialists on clinical indicators for identification and management of advanced Parkinson's disease: a multi-country Delphi-panel approach. Curr. Med. Res. Opin. 34(12), 2063-2073 (2018). 
5. Titova N, Chaudhuri KR. Personalized medicine in Parkinson's disease: time to be precise. Mov. Disord. 32(8), 1147-1154 (2017).

6. Olanow CW, Kieburtz K, Odin P et al. Continuous intrajejunal infusion of levodopa-carbidopa intestinal gel for patients with advanced Parkinson's disease: a randomised, controlled, double-blind, double-dummy study. Lancet Neurol. 13(2), 141-149 (2014).

7. Slevin JT, Fernandez HH, Zadikoff C et al. Long-term safety and maintenance of efficacy of levodopa-carbidopa intestinal gel: an open-label extension of the double-blind pivotal study in advanced Parkinson's disease patients. J. Parkinsons Dis. 5(1), 165-174 (2015).

8. Fernandez HH, Boyd JT, Fung VSC et al. Long-term safety and efficacy of levodopa-carbidopa intestinal gel in advanced Parkinson's disease. Mov. Disord. 33(6), 928-936 (2018).

9. Fernandez HH, Standaert DG, Hauser RA et al. Levodopa-carbidopa intestinal gel in advanced Parkinson's disease: final 12-month, open-label results. Mov. Disord. 30(4), 500-509 (2015).

10. Antonini A, Poewe W, Chaudhuri KR et al. Levodopa-carbidopa intestinal gel in advanced Parkinson's: final results of the GLORIA registry. Parkinsonism Relat. Disord. 45, 13-20 (2017).

- The GLORIA registry evaluated long-term levodopa-carbidopa intestinal gel (LCIG) efficacy in 375 patients in a routine clinical setting. LCIG use was associated with significant reductions in 'Off' time and 'On' time with dyskinesia over 24 months of observation.

11. Standaert DG, Rodriguez RL, Slevin JT et al. Effect of levodopa-carbidopa intestinal gel on non-motor symptoms in patients with advanced Parkinson's disease. Mov Disord Clin Pract. 4(6), 829-837 (2017).

12. Lopiano L, Modugno N, Marano P et al. Motor and non-motor outcomes in patients with advanced Parkinson's disease treated with levodopa/carbidopa intestinal gel: final results of the GREENFIELD observational study. J. Neurol. 266(9), 2164-2176 (2019).

- The GREENFIELD observational study examined real-world LCIG effects on motor symptoms, nonmotor symptoms and patient and caregiver quality of life in 145 patients.

13. Honig H, Antonini A, Martinez-Martin P et al. Intrajejunal levodopa infusion in Parkinson's disease: a pilot multicenter study of effects on nonmotor symptoms and quality of life. Mov. Disord. 24(10), 1468-1474 (2009).

14. Dafsari HS, Martinez-Martin P, Rizos A et al. EuroInf 2: subthalamic stimulation, apomorphine and levodopa infusion in Parkinson's disease. Mov. Disord. 34(3), 353-365 (2019).

15. Kruger R, Lingor P, Doskas $\mathrm{T}$ et al. An observational study of the effect of levodopa-carbidopa intestinal gel on activities of daily living and quality of life in advanced Parkinson's disease patients. Adv. Ther. 34(7), 1741-1752 (2017).

16. Antonini A, Moro E, Godeiro C, Reichmann H. Medical and surgical management of advanced Parkinson's disease. Mov. Disord. 33(6), 900-908 (2018).

-• Outlining a standardized definition of advanced Parkinson's disease (APD) is important for anticipating patient outcomes as PD progresses. This Delphi expert consensus panel outlines the development of the 5-2-1 criteria for identifying patients with advanced Parkinson's disease.

17. Morgante L, Basile G, Epifanio A et al. Continuous apomorphine infusion (CAI) and neuropsychiatric disorders in patients with advanced Parkinson's disease: a follow-up of two years. Arch Gerontol Geriatr Suppl. doi:10.1016/j.archger.2004.04.039(9), 291-296 (2004) (Epub ahead of print).

18. Todorova A, Samuel M, Brown RG, Chaudhuri KR. Infusion therapies and development of impulse control disorders in advanced Parkinson disease: clinical experience after 3 years' follow-up. Clin. Neuropharmacol. 38(4), 132-134 (2015).

19. Katzenschlager R, Poewe W, Rascol O, Trenkwalder G. Double-blind, randomized, placebo-controlled, phase III study (TOLEDO) to evaluate the efficacy of apomorphine subcutaneous infusion in reducing OFF time in Parkinson's disease patients with motor fluctuations not well controlled on optimized conventional treatment. Mov. Disord. 32(Suppl. 2), S518-S519 (2017).

20. Obeso JA, Olanow CW, Rodriguez-Oroz MC, Krack P, Kumar R, Lang AE. Deep-brain stimulation of the subthalamic nucleus or the pars interna of the globus pallidus in Parkinson's disease. N. Engl. J. Med. 345(13), 956-963 (2001).

21. Deuschl G, Schade-Brittinger C, Krack P et al. A randomized trial of deep-brain stimulation for Parkinson's disease. N. Engl. J. Med. 355(9), 896-908 (2006).

22. Follett KA, Weaver FM, Stern M et al. Pallidal versus subthalamic deep-brain stimulation for Parkinson's disease. N. Engl. J. Med. 362(22), 2077-2091 (2010).

23. Weaver FM, Follett K, Stern M et al. Bilateral deep brain stimulation vs best medical therapy for patients with advanced Parkinson disease: a randomized controlled trial. JAMA 301(1), 63-73 (2009).

24. Odekerken VJ, Van Laar T, Staal MJ et al. Subthalamic nucleus versus globus pallidus bilateral deep brain stimulation for advanced Parkinson's disease (NSTAPS study): a randomised controlled trial. Lancet Neurol. 12(1), 37-44 (2013).

25. Schuepbach WM, Rau J, Knudsen K et al. Neurostimulation for Parkinson's disease with early motor complications. N. Engl. J. Med. 368(7), 610-622 (2013).

26. Martinez-Martin P, Reddy P, Katzenschlager R et al. EuroInf: a multicenter comparative observational study of apomorphine and levodopa infusion in Parkinson's disease. Mov. Disord. 30(4), 510-516 (2015).

27. Coelho M, Ferreira JJ. Late-stage Parkinson disease. Nat. Rev. Neurol. 8(8), 435-442 (2012). 
28. Chaudhuri KR, Schapira AH. Non-motor symptoms of Parkinson's disease: dopaminergic pathophysiology and treatment. Lancet Neurol. 8(5), 464-474 (2009).

29. Fox SH, Katzenschlager R, Lim SY et al. The Movement Disorder Society evidence-based medicine review update: treatments for the motor symptoms of Parkinson's disease. Mov. Disord. 26(Suppl. 3), S2-S41 (2011)

30. Cabrera LY, Sarva H, Sidiropoulos C. Perspectives on the earlier use of deep brain stimulation for Parkinson disease from a qualitative study of U.S. clinicians. World Neurosurg. doi:10.1016/j.wneu.2019.03.051 (2019) (Epub ahead of print).

- This survey of clinicians examines deep brain stimulation referral and use and found that clinicians considered earlier use based on patient symptoms. However, the criteria clinicians used for referrals varied.

31. Fasano A, Fung VSC, Lopiano L et al. Characterizing advanced Parkinson's disease: OBSERVE-PD observational study results of 2615 patients. BMC Neurol. 19(1), 50 (2019).

-• OBSERVE PD was an international observational study that aims to characterize advanced Parkinson's disease by examining characteristics of patients classified as having advanced Parkinson's disease as assessed by an experienced physician.

32. Antonini A, Odin P, Jalundhwala YJ, Schmidt P, Skalicky AM. MANAGE-PD: a clinician-reported tool to identify patients with Parkinson's disease inadequately controlled on oral medications-results from vignette-based validation. World Congress on Parkinson's Disease and Related Disorders, Montreal, QC, Canada (2019).

-. Making Informed Decisions to Aid Timely Management of Parkinson's Disease (MANAGE-PD) is a screening tool developed by a panel of movement disorder specialists that is designed to support decision-making for PD symptom management.

33. Odin P, Chaudhuri KR, Volkmann J et al. Viewpoint and practical recommendations from a movement disorder specialist panel on objective measurement in the clinical management of Parkinson's disease. NPJ Parkinsons Dis. 4, 14 (2018).

- Consensus guidelines on objective measurements and related devices for use by clinicians in Parkinson's disease treatment. These guidelines focus on defining a cutoff for 'controlled' and 'uncontrolled' symptoms.

34. Martinez-Martin P, Rodriguez-Blazquez C, Mario A et al. Parkinson’s disease severity levels and MDS-Unified Parkinson's Disease Rating Scale. Parkinsonism Relat. Disord. 21(1), 50-54 (2015).

35. Brown RG, Dittner A, Findley L, Wessely SC. The Parkinson fatigue scale. Parkinsonism Relat. Disord. 11(1), 49-55 (2005).

36. Chaudhuri KR, Martinez-Martin P, Brown RG et al. The metric properties of a novel non-motor symptoms scale for Parkinson's disease: results from an international pilot study. Mov. Disord. 22(13), 1901-1911 (2007).

37. Hoehn MM, Yahr MD. Parkinsonism: onset, progression and mortality. Neurology 17(5), 427-442 (1967). 


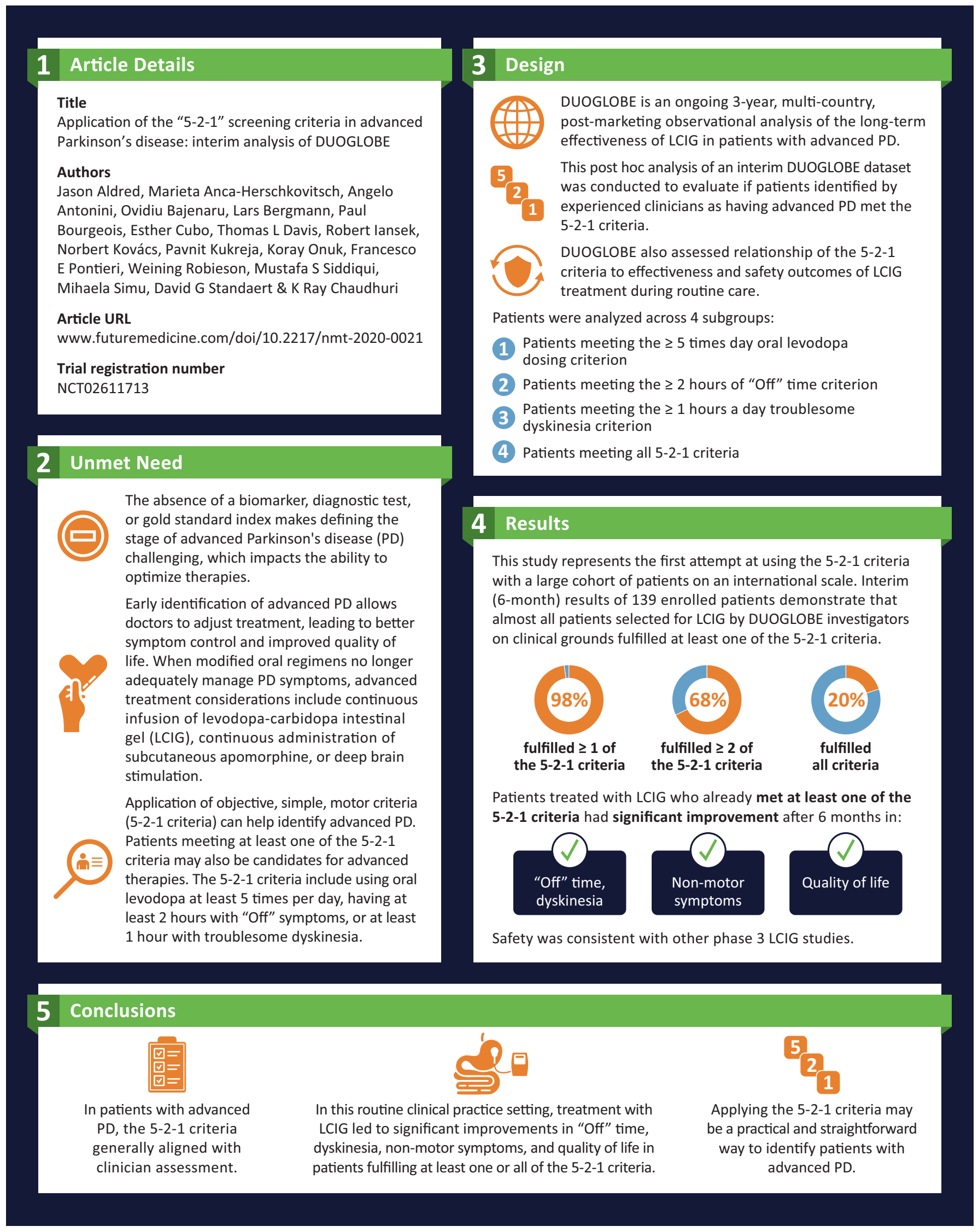

